\title{
A comparison of the acute effects of radial extracorporeal shockwave therapy, ultrasound therapy, and exercise therapy in plantar fasciitis
}

\author{
Bihter Akınoğlu ${ }^{1, *}$, Nezire Köse ${ }^{2}$ \\ ${ }^{1}$ Ankara Yıldırım Beyazıt University, Faculty of Health Sciences, Department of Physiotherapy and Rehabilitation, Ankara, Turkey \\ ${ }^{2}$ Hacettepe University, Faculty of Health Sciences, Department of Physiotherapy and Rehabilitation, Ankara, Turkey
}

This study was conducted to compare the acute effects of radial extracorporeal shockwave therapy (r-ESWT), ultrasound (US) therapy and home exercise therapy on pain, fatigue, performance and walking distance in women with plantar fasciitis (PF). Fifty-four female patients diagnosed with PF participated in the study. The patients were randomly divided into 3 groups as r-ESWT and home exercise groups, US and home exercise groups, and only home exercise groups. Patients' pain and fatigue levels were assessed by using a visual analog scale. Also walking distances, walking speed and plantar flexor muscles performance were assessed. Patients' pain at night before bedtime and leg fatigue after the 6-min walking test decreased in the US therapy group in comparison to the other two groups $(P<0.05)$. The 20 -m walking speed improved in the US and r-ESWT therapy groups following the treatments $(P<0.05)$ and there was no difference between the groups. After treatment, while the 20-m walking speed was increasing in US and $r$-ESWT groups $(P<0.05)$, no increase was observed in exercise group. But there was no difference between the 20-m walking speed of 3 groups after treatment $(P>0.05)$. However, all three groups' complaints decreased after treatment. Our study results illustrated that $r$-ESWT, US, and home exercise therapy have acute effects on the PF treatment and when there is no possibility of practising any treatment to patients, home exercises such as r-ESWT, US treatment are effective therapies for decreasing pain and fatigue levels and improving performance and walking distance.

Keywords: Exercise, Physiotherapy, Plantar fasciitis

\section{INTRODUCTION}

Plantar fasciitis (PF) is a musculoskeletal system disorder and it is seen on feet most frequently and affects plantar fascia especially (Huang et al., 2010; Sahin et al., 2010). Plantar fascia is the major stabilizer of the longitudinal arch. Therefore, the factors which disrupt the normal biomechanics of feet increase plantar fascia tension. Abnormal tension of plantar fascia that is caused by biomechanical factors does not lead to clinical symptoms at the beginning. However, the stress-causing factors including running, instantly increased activity level, obesity and rapid weight gain, nonorthopedic shoes, long-term standing up or walking and doing activities on a hard surface cause plantar fascia and repetitive microtraumas in other structures and lead to clinical symptoms
(Wearing et al., 2007). In case this situation is not treated, soft tissue calcification starts as a result of microlacerations and thus, calcification and degenerative changes (PF) occur (Lee et al., 2009; Tounton et al., 2002).

The major complaints of patients with PF are stinging and burning pain sensed inside the heel and especially in the heel medial. Pain is excessive at the first steps after getting up in the morning. It alleviates after a few steps however it becomes severe towards the end of the day based on activity. Pain moderates during resting however after the resting it restarts at the first step. Sometimes, slight swelling and erythema occurs. The symptom period may range from several weeks to years (Pfeffer, 1995).

The treatment-means for PF are divided into surgical and conservative methods. The conservative treatments consist of resting,
${ }^{*}$ Corresponding author: Bihter Akınoğlu (D) https://orcid.org/0000-0002-8214-7895 Ankara Yildırım Beyazıt University, Faculty of Health Sciences, Department of Physiotherapy and Rehabilitation, Ankara, Turkey

Tel: +90-5059384136, Fax: +90-3123051515, E-mail: rgkardelen@yahoo.com

Received: January 23, 2018 / Accepted: March 10, 2018
This is an Open Access article distributed under the terms of the Creative Commons Attribution Non-Commercial License (http://creativecommons.org/licenses/by-nc/4.0/) which permits unrestricted non-commercial use, distribution, and reproduction in any medium, provided the original work is properly cited. 
soft sole supports, heel elevation, night braces keeping the plantar fascia tense at the dorsiflexion, plaster casts, orthosis-type shoe wearing, acupuncture, ultrasound, iontophoresis methods, low density laser application, extracorporeal shockwave therapy, stretching and strengthening exercises, anti-inflammatory (nonsteroid and steroid) medications, cortisone injections and botulinum toxin injection (Cacchio et al., 2006; Cotchett et al., 2014; Covey and Mulder, 2013; Díaz López and Guzmán Carrasco, 2014; Greve et al., 2009; Huang et al., 2010; Lee et al., 2009; Tsai et al., 2010; Vahdatpour et al., 2012). The conservative therapy is successful 90\%-95\%. Therefore, the surgical method must be resorted to as an ultimate choice (Cotchett et al., 2014; Covey and Mulder, 2013). Studies are conducted comparing various treatment methods in order to find the most effective therapy for PF (Greve et al., 2009; Jha et al., 2013; Lee et al., 2009; Pienimaki et al., 1996; Ryan et al., 2014; Vahdatpour et al., 2012; Yüzer et al., 2006). It was stated that more detailed studies must be conducted on this subject (Greve et al., 2009). Hence, our study was planned to compare the effects of r-ESWT and US therapies and home exercise program for $\mathrm{PF}$ treatment.

\section{MATERIALS AND METHODS}

\section{Participants and settings}

Eighty-eight female patients were called for the study who applied to our clinic of Physical Medicine and Rehabilitation with heel pain during January 2013 and December 2014 and had heel sensitivity detected by the physical examination with palpation, and had calcaneal spur as seen in feet-lateral grapy and were diagnosed with PF in a single foot. The study was based on volunteerism and the inclusion of the study are; had longer than 3 months of disease duration, were not treated by any physical therapy method previously, received no local steroid injection and did not undergo any surgical procedure, not doing regular exercise and not using any special kind if shoes. The study was started with 78 female patients by leaving 10 patients out of the study due to reflected pain because of their lower extremity pathologies, pregnancy, hemorrhage, neurologic deficit, malignity, using cardiac pacemaker or inflammatory illness story and anticoagulant use. After the first assessment of the patients who complied with the study criteria, the patients were divided randomly into 3 groups namely the r-ESWT+exercise group, the US+exercise group and the exercise group. Randomization was performed by sealed envelope method. As a result of randomization, there were 24 patients in the r-ESWT+exercise group, 26 patients in the US+exercise group and 28 patients in the exercise group. Two patients in the r-ESWT+exercise group left the study because they could not stand pain, two patients were dropped from the study because they did not follow the exercise program and two patients were dropped from the study because they did not continue the treatment. Three patients in the US group left the study because they had the same symptoms starting in their other leg, three patients were dropped from the study because they did not come to the treatment and two patients were dropped from the study because they did not follow the exercise program. Six patients in the exercise group were dropped from the study because they expressed in their follow-up appointment that they did not do their exercises on a regular basis and four patients were dropped from the study because they did not show up for their follow up appointment. Hence, our study was conducted on 54 patients (their average age was 50 and their distribution was 39-59).

In order to conduct the study, the approval and the written, clarified consents of the participants were taken (GO13/218).

The patients were informed about the planned procedure and informed patient consents were received. The study was conducted in accord with Helsinki Declaration. Similar studies were used and an effort analysis was made to designate the patient number prior to the study. The minimum patient number was estimated as 16 for each group with the type I error of $\alpha=0.05$ and the type II error of $\beta=0.2$ and with a $95 \%$ confidence interval and \pm 2 standard deviation (SD).

The patients were informed about the study and informed patient consents were received. At the beginning, the demographic data of all patients, their pain levels in the morning, at night and after the 6-min walking test, their leg fatigue levels after the 6-min walking test and their general fatigue levels were measured by using the visual analogy scale (VAS). Their walking distances were assessed by using the 6-min walking test. Furthermore, the flexor muscle performances of the patients were assessed by using the heel-rise test and their walking speeds were assessed by using the $20-\mathrm{m}$ walking test. These tests were assessed prior to the randomization and at the end of the therapies ( 4 weeks after the first treatment).

\section{Treatment protocols $r$-ESWT therapy}

The patients in the first group were treated by Swiss Dolor Clast brand device of the Electro medical Systems SA (EMS) Company (Nyon, Switzerland). The patients were treated as they were lying in prone position. Prior to starting the therapy sessions, the pa- 
tients' most painful 5 points were uncovered by palpation and marked by a pen. A total of 1,500 pulses of the r-ESWT therapy was applied to each of the five painful points as $0.2 \mathrm{mj} / \mathrm{mm}^{2}, 3.0 \mathrm{~Hz}$ of 500 pulses; $0.3 \mathrm{mj} / \mathrm{mm}^{2}, 8 \mathrm{~Hz}$ of 300 pulses were applied to the entire heel location and a total of 2,000 pulses of the r-ESWT therapy was applied in each session. Gel was used between the cap and skin during the applications for ensuring conductivity. The therapy was applied in 3 sessions once a week (Yörük and Kırd, 2014).

\section{US therapy}

It was applied to the patients in the second group by using Gymna Pulson 200 device. The therapy was applied as the patients were lying in prone position and by longitudinal movements along the entire plantar fascia. The US therapy was applied at 3.0-MHz frequency and as $20 \%$ intermittently with $1 \mathrm{~W} / \mathrm{cm}^{2}$ of power for $8 \mathrm{~min}$. Gel was used between the US cap and skin during the applications for ensuring conductivity (Robertson and Baker, 2001). The patients were treated in a total 7 sessions 2 days a week (to equate treatment time with r-ESWT therapy).

\section{The exercise program}

Home exercise programs were applied in all three groups of patients. The patients were trained on the exercise program and subsequently provided with an exercise form for marking the exercise dates and sessions. The patients were asked to do these exercises 10 times by counting up to 30 for a total of 4 weeks in the mornings and at nights starting from the first assessment until the second assessment. The home exercise program consisted of Achilles tendon stretching by sitting, gastrocnemius and gastrosoleus muscles stretching as standing up and plantar fascia stretching on steps (Digiovanni et al., 2006).

\section{Statistical analysis}

The data were analyzed by SPSS ver. 15.0 (SPSS Inc., Chicago, IL, USA) and via descriptive statistics analysis (frequency, mean, minimum and maximum, and SD). Before starting the study, a power analysis was performed to determine the number of patients required. The Kolmogorov-Smirnov test was used to determine whether data distribution was normal, and it was determined that the data did not have a normal distribution. We therefore used the Wilcoxon signed rank test to compare the treatment groups' data before and after the treatment. The Kruskal-Wallis test was used to determine the group causing the differences between the three groups. The Mann-Whitney $U$-test was used to make comparisons between the two groups. The statistical significance level was a $P$-value of less than 0.05 , and we used $95 \%$ confidence intervals.

\section{RESULTS}

There were no differences found between the demographic features of the groups including their educational status and body mass indexes (BMIs) $(P>0.05)$ (Table 1 ).

The values of the participants prior to the therapies are shown in Table 1. The values of pain before the therapies, fatigue after a 6-min walk, 6-min walking distance, heel-rise test scores and $20-\mathrm{m}$ walking speed were found similar in the three groups $(P>0.05)$ (Table 2).

The pain and fatigue levels decreased following the therapies in the three groups and their 6-min walking distances and heel rise test scores increased $(P<0.05)$. The $20-\mathrm{m}$ walking speed increased in the US and r-ESWT groups $(P<0.05)$ following the therapies and there was no different found in the Ex group $(P>0.05)$ (Table 3).

By comparing the groups with each other following the therapies, pain before bedtime at nights and leg fatigue after a 6-min walk decreased more in the US group $(P<0.05)$. The groups were similar in terms of morning pain, pain after a 6-min walk, fatigue after a 6-min walk $(P>0.05)$. Also 6-min walking distance, heel rise test scores and 20-min walking speed were similar in three groups $(P>0.05)$ (Table 4$)$.

Table 1. Patient demographics

\begin{tabular}{lccccc}
\hline Variable & r-ESWT group $(\mathrm{n}=18)$ & US group $(\mathrm{n}=18)$ & Ex group $(\mathrm{n}=18)$ & Chi-squre $^{\text {a) }}$ & $P_{\text {-value }}$ \\
\hline Age $(\mathrm{yr})$ & $50.00 \pm 6.54(39-59)$ & $50.11 \pm 9.29(32-65)$ & $45.22 \pm 7.64(32-62)$ & 4.596 & 0.100 \\
Education level $(\mathrm{yr})$ & $3.94 \pm 3.75(1-11)$ & $3.22 \pm 4.04(0-11)$ & $3.78 \pm 4.02(0-11)$ & 3.281 & 0.703 \\
Body mass index $\left(\mathrm{kg} / \mathrm{m}^{2}\right)$ & $28.58 \pm 1.67(23.51-29.94)$ & $28.48 \pm 2.15(21.97-29.94)$ & $28.03 \pm 2.04(22.95-29.97)$ & 1.299 & 0.522 \\
\hline
\end{tabular}

Values are presented as mean \pm standard deviation (range).

$r$-ESWT, radial extracorporeal shock wave therapy; US, ultrasound therapy; Ex, exercise therapy.

${ }^{a}$ Kruskal-Wallis test. 
Table 2. Patients' pretreatment evaluation results

\begin{tabular}{|c|c|c|c|c|c|}
\hline Before treatment & $r$-ESWT group & US group & Ex group & Chi-squre ${ }^{\text {al }}$ & $P$-value \\
\hline \multicolumn{6}{|l|}{ Pain (cm) } \\
\hline Morning pain & $9.33 \pm 1.41$ & $9.50 \pm 1.29$ & $8.50 \pm 2.55$ & 1.182 & 0.554 \\
\hline Pain before bedtime & $9.00 \pm 1.75$ & $8.83 \pm 1.86$ & $9.06 \pm 1.80$ & 0.147 & 0.929 \\
\hline Pain after 6-min walk test & $3.78 \pm 3.44$ & $8.17 \pm 2.18$ & $7.39 \pm 1.97$ & 4.704 & 0.095 \\
\hline \multicolumn{6}{|l|}{ Fatique after 6-min walk test (cm) } \\
\hline General fatique & $6.94 \pm 2.53$ & $5.22 \pm 3.42$ & $4.83 \pm 3.03$ & 5.670 & 0.059 \\
\hline Leg fatique & $6.72 \pm 2.35$ & $4.11 \pm 3.80$ & $4.28 \pm 3.82$ & 5.079 & 0.079 \\
\hline Distance of 6-min walk test (m) & $366 \pm 94$ & $359 \pm 88$ & $400 \pm 58.21$ & 3.273 & 0.195 \\
\hline Heel rise test score (pcs/min) & $9.00 \pm 6.53$ & $10.89 \pm 5.79$ & $10.00 \pm 6.36$ & 1.041 & 0.594 \\
\hline 20-m walk speed (sn) & $10.72 \pm 3.06$ & $11.28 \pm 3.29$ & $9.78 \pm 2.07$ & 2.156 & 0.340 \\
\hline
\end{tabular}

Values are presented as mean \pm standard deviation.

r-ESWT, radial extracorporeal shock wave therapy; US, ultrasound therapy; Ex, exercise therapy.

${ }^{a}$ Kruskal-Wallis test.

Table 3. Comparision of the groups' results before and after treatment

\begin{tabular}{|c|c|c|c|c|c|c|c|c|c|c|c|c|}
\hline \multirow{2}{*}{ Variable } & \multicolumn{4}{|c|}{ r-ESWT group } & \multicolumn{4}{|c|}{ US group } & \multicolumn{4}{|c|}{ Ex group } \\
\hline & BT & AT & Z & $P$-value & BT & AT & Z & $P$-value & BT & AT & $Z$ & $P$-value \\
\hline \multicolumn{13}{|l|}{ Pain (cm) } \\
\hline Morning & $9.33 \pm 1.41$ & $4.94 \pm 4.12$ & -3.304 & 0.001 & $9.50 \pm 1.29$ & $3.72 \pm 2.47$ & -3.754 & 0.001 & $8.50 \pm 2.55$ & $5.61 \pm 3.58$ & -2.871 & 0.004 \\
\hline Before bedtime & $9.00 \pm 1.75$ & $5.94 \pm 3.64$ & -2.809 & 0.005 & $8.83 \pm 1.86$ & $3.89 \pm 2.35$ & -3.530 & 0.001 & $9.06 \pm 1.80$ & $6.39 \pm 2.75$ & -2.821 & 0.005 \\
\hline After 6-min walk test & $3.78 \pm 3.44$ & $3.78 \pm 3.44$ & -3.366 & 0.001 & $8.17 \pm 2.18$ & $3.17 \pm 3.37$ & -3.303 & 0.001 & $7.39 \pm 1.97$ & $4.83 \pm 2.62$ & -3.079 & 0.002 \\
\hline \multicolumn{13}{|l|}{ Fatique after 6-min walk test (cm) } \\
\hline General fatique & $6.94 \pm 2.53$ & $3.72 \pm 3.18$ & -3.195 & 0.001 & $5.22 \pm 3.42$ & $1.94 \pm 2.36$ & -2.941 & 0.003 & $4.83 \pm 3.03$ & $2.89 \pm 2.97$ & -1.799 & 0.072 \\
\hline Leg fatique & $6.72 \pm 2.35$ & $3.72 \pm 2.85$ & -3.045 & 0.002 & $4.11 \pm 3.80$ & $1.28 \pm 1.81$ & -2.558 & 0.011 & $4.28 \pm 3.82$ & $2.72 \pm 3.56$ & -1.476 & 0.140 \\
\hline Distance of 6-min walk test (m) & $366 \pm 94$ & $486 \pm 112$ & -3.413 & 0.001 & $359 \pm 88$ & $473 \pm 144$ & -3.420 & 0.001 & $400 \pm 58.21$ & $455 \pm 92$ & -2.352 & 0.019 \\
\hline Heel rise test score (pcs/min) & $9.00 \pm 6.53$ & $15.83 \pm 5.25$ & -3.520 & 0.001 & $10.89 \pm 5.79$ & $18.72 \pm 8.93$ & -2.899 & 0.004 & $10.00 \pm 6.36$ & $18.11 \pm 7.37$ & -3.466 & 0.001 \\
\hline 20-m walk speed (sn) & $10.72 \pm 3.06$ & $8.33 \pm 1.72$ & -3.457 & 0.001 & $11.28 \pm 3.29$ & $8.72 \pm 2.72$ & -3.421 & 0.001 & $9.78 \pm 2.07$ & $8.67 \pm 1.94$ & -1.862 & 0.063 \\
\hline
\end{tabular}

Values are presented as mean \pm standard deviation. Wilcoxon signed ranks test.

r-ESWT, radial extracorporeal shock wave therapy; US, ultrasound therapy; Ex, exercise therapy; BT, before treatment; AT, after treatment.

Table 4. Patients' posttreatment evaluation results

\begin{tabular}{|c|c|c|c|c|c|}
\hline After treatment & $r$-ESWT group & US group & Ex group & Chi-squre ${ }^{c)}$ & $P$-value \\
\hline \multicolumn{6}{|l|}{ Pain (cm) } \\
\hline Morning pain & $4.94 \pm 4.12$ & $3.72 \pm 2.47$ & $5.61 \pm 3.58$ & 2.566 & 0.277 \\
\hline Pain before bedtime & $5.94 \pm 3.64$ & $3.89 \pm 2.35^{\mathrm{a}, \mathrm{b})}$ & $6.39 \pm 2.75^{b /}$ & 6.922 & 0.031 \\
\hline Pain after 6-min walk test & $3.78 \pm 3.44$ & $3.17 \pm 3.37$ & $4.83 \pm 2.62$ & 2.677 & 0.262 \\
\hline \multicolumn{6}{|l|}{ Fatique after 6-min walk test (cm) } \\
\hline General fatique & $3.72 \pm 3.18$ & $1.94 \pm 2.36$ & $2.89 \pm 2.97$ & 3.214 & 0.200 \\
\hline Leg fatique & $3.72 \pm 2.85^{a)}$ & $1.28 \pm 1.81^{\mathrm{a}, \mathrm{bl}}$ & $2.72 \pm 3.56$ & 6.289 & 0.043 \\
\hline Distance of 6-min walk test ( $\mathrm{m})$ & $486 \pm 112$ & $473 \pm 144$ & $455 \pm 92$ & 0.352 & 0.839 \\
\hline Heel rise test score (pcs/min) & $15.83 \pm 5.25$ & $18.72 \pm 8.93$ & $18.11 \pm 7.37$ & 1.254 & 0.534 \\
\hline 20-m walk speed (sn) & $8.33 \pm 1.72$ & $8.72 \pm 2.72$ & $8.67 \pm 1.94$ & 0.161 & 0.923 \\
\hline
\end{tabular}

Values are presented as mean \pm standard deviation.

$r$-ESWT, radial extracorporeal shock wave therapy; US, ultrasound therapy; Ex, exercise therapy.

alAccording to Mann Whitney U-test, there is difference between the r-ESWT vs. US groups. ${ }^{b}$ According to Mann Whitney U-test, there is difference between the US and Control groups. ${ }^{c}$ Kruskal-Wallis test. 


\section{DISCUSSION}

In our study, we aimed to assess the acute effects of the r-ESWT, US, and exercise therapies for PF immediately after the therapies and we formed three groups namely the r-ESWT and exercise groups, the US and exercise groups and only the exercise group. At the end of the study, it was designated that the patients in all three groups got better after the therapies primarily the patients in the US group. In this group, pain before bedtime at nights and leg fatigue after a 6-min walk diminished more in comparison to the other groups.

$\mathrm{PF}$ is the most frequent cause of heel pain. Despite the conservative methods are used during therapies including steroid injection, nonsteroid anti-inflammatory medication, iontophoresis, laser, ultrasound, ESWT, orthotic support therapy and exercise therapies (Greve et al., 2009; Metzner et al., 2010; Tsai et al., 2010; Yüzer et al., 2006), there is no consensus about the most successful treatment method (Rompe, 2002; Tounton et al., 2002). Therefore, our study is unique since we organized it to compare the effects of r-ESWT and US therapies applied in combination with the exercise therapy for PF treatment and included a group doing only home exercises.

The descriptive features of our cases including age, BMI, education and occupation status, and dominant leg activations were similar in the three groups and we think that they may affect our assessments in our study, exercise program implementation and the therapy success. This showed us that we conducted our study on homogenous groups.

Another result of our study indicating to the homogeneity of the groups was that pain before the therapies, heel rise test scores, 6-min walking distance, leg fatigue level after a 6-min walk and general fatigue level and 20-m walking speed results were similar in the three groups.

Pain is the major complaint of patients with PF. Pain on medial calcaneal tubercle is generally unbearable in the first step taken in the morning and this pain moderates somewhat after a few steps and increase again after long-term activity and before bedtime at nights (Lee et al., 2009). As a result of our study, morning pain, pain before bedtime at nights and pain after the 6-min walking test decreased in the three groups after the therapies. Pain before bedtime at nights moderated more in the US group. This showed us that all three therapy methods were effective to decrease pain however the ultrasound therapy was even more influential for moderating pain that increase with activity. Upon the literature search, there were no studies found comparing the effects of r-ES-
WT and US therapies on pain however in the literature, the studies conducted on the effects of the ESWT, US, and exercise applications on pain reported that these practices were effective on pain moderation (Bolívar et al., 2013; Ryan et al., 2014; Szabó et al., 2010; Tounton et al., 2002; Vahdatpour et al., 2012; Yüzer et al., 2006) and these results were parallel to our study.

Another parameter assessed in our study was fatigue. The general fatigue levels of the patients and their leg fatigue after the 6-min walking test were assessed by using the VAS and it was uncovered that the fatigue levels of the three groups went down after the therapies. However, it was exhibited that their leg fatigues after the 6-min walking test alleviated even more in the US group in comparison to the other groups. This made us think that this situation occurred because the pain that escalated with activity was more moderate in the US group in comparison to the other two groups.

Walking is one of the activities affected in PF. In our study, the 6-min walking test distances were assessed and found that this distance increased in all of the three groups after the therapies and there were no difference between the groups. Dastgir (2014) studied the effects of the ESWT therapy in patients with PF and found that the patients' painless walking distances increased in parallel to our results.

Heel rising is a frequently used activity in daily life and at the same time it is a phase of walking and patients with PF have difficulty doing it since plantar fascia stretches during this activity. Individuals may face difficulty during heel lifting and due to pain inflicted during walking and their walking speed also may slow down. We assessed the heel-rise test scores because of these reasons and despite the patients' heel-rise test scores and 20-m walking speeds were similar prior to the therapies, they increased in all of the three groups after the therapies and there were no differences found between the groups. In the literature, there were no studies found conducted on walking speeds of patients with PF. Furthermore, there were no studies found investigating the effects of the applied therapies on heel rise and walking speed either. However, there are studies investigating the effects of pain on walking distance and functionality in various patient groups and reporting that as the pain severity increased, the individuals' walking speeds and functionality levels dropped (Bohannon, 1997; Mänty et al., 2014). As a result of our study, in parallel to this information, we think that the increased heel-rise test scores and walking speeds of the patients stem from their moderated pain.

Considering our literature search about the parameters that we applied generally, despite there are many studies on ESWT and r-ESWT in PF, there is no consensus reached about the therapy 
protocol as far as the pulse number, pressure and application frequency are concerned. It is difficult to make a comparison between the studies since various tools were used and different protocols were applied in the studies (Sems et al., 2006). Stasinopoulos and Johnson (2005) reported as a result of their study that the effects of shockwaves emerged based on dosage and an appropriate dose was not designated yet. It was thought that the diversity of patient population and the variation of applied dosages influenced the evidence level in the US therapy just like in the ESWT and therefore the most appropriate dose was not able to be designated (Robertson and Baker, 2001). Moreover, the superiority of stretching exercises to one another was researched and considering their short-term results, plantar fascia stretching exercises were reported to be more effective in comparison to the Achilles tendon stretching exercises however it was pointed out that the stretching exercises must be combined (Jha et al., 2013; Sweeting et al., 2011). The review studies conducted on patients with PF showed that a single therapy modality for PF treatment was generally inadequate and more than one therapy modality must be used in combination. In our study, we concluded that the US therapy combined with exercises and the r-ESWT therapy combined with exercises were more successful on some parameters such as walking speed in comparison to the exercise therapy alone. However, we recommend that home exercise programs must be applied for patients with PF, whose treatment is not possible in a clinic environment since it would moderate patients' pain and improve their functionality, walking speed and walking distance.

This study had some limitations. Our limitations were using of subjective measures and not having control group without doing home exercise and the lack of therapy groups for whom no home exercise program was applied.

In conclusion, as a result of our study it was seen that the exercise therapy, and the US therapy and the r-ESWT therapy applied in combination with the exercise therapy were effective methods for moderating pain and improving walking distance, walking speed and functionality. It was seen that the US+exercise therapy for patients with PF was more superior than the exercise therapy, and the r-ESWT therapy combined with the exercise therapy for moderating pain before bedtime at nights. Nevertheless, it was concluded that further studies needed to be conducted for comparing the applications of the r-ESWT and US therapies performed with various dosages, diagnoses, and consisting of a combination of more different therapy modalities and using wider samplings.

\section{CONFLICT OF INTEREST}

No potential conflict of interest relevant to this article was reported.

\section{REFERENCES}

Bohannon RW. Comfortable and maximum walking speed of adults aged 20-79 years: reference values and determinants. Age Ageing 1997;26: 15-19.

Bolívar YA, Munuera PV, Padillo JP. Relationship between tightness of the posterior muscles of the lower limb and plantar fasciitis. Foot Ankle Int 2013;34:42-48.

Cacchio A, Paoloni M, Barile A, Don R, de Paulis F, Calvisi V, Ranavolo A, Frascarelli M, Santilli V, Spacca G. Effectiveness of radial shock-wave therapy for calcific tendinitis of the shoulder: single-blind, randomized clinical study. Phys Ther 2006;86:672-682.

Cotchett MP, Munteanu SE, Landorf KB. Effectiveness of trigger point dry needling for plantar heel pain: a randomized controlled trial. Phys Ther 2014;94:1083-1094.

Covey CJ, Mulder MD. Plantar fasciitis: How best to treat? J Fam Pract 2013;62:466-471.

Dastgir N. Extracorporeal shock wave therapy for treatment of plantar fasciitis. J Pak Med Assoc 2014;64:675-678

Díaz López AM, Guzmán Carrasco P. Effectiveness of different physical therapy in conservative treatment of plantar fasciitis: systematic review. Rev Esp Salud Publica 2014;88:157-178.

Digiovanni BF, Nawoczenski DA, Malay DP, Graci PA, Williams TT, Wilding GE, Baumhauer JF. Plantar fascia-specific stretching exercise improves outcomes in patients with chronic plantar fasciitis. A prospective clinical trial with two-year follow-up. J Bone Joint Surg Am 2006;88:1775-1781.

Greve JM, Grecco MV, Santos-Silva PR. Comparison of radial shockwaves and conventional physiotherapy for treating plantar fasciitis. Clinics (Sao Paulo) 2009;64:97-103.

Huang YC, Wei SH, Wang HK, Lieu FK. Ultrasonographic guided botulinum toxin type A treatment for plantar fasciitis: an outcome-based investigation for treating pain and gait changes. J Rehabil Med 2010;42: 136-140.

Jha RK, Uprety S, Shah LL. Functional outcome in patients with chronic plantar fasciitis treated with plantar fascia stetching vs tendoachilles stretching exercises. J Inst Med 2013;35:32-38.

Lee SY, McKeon P, Hertel J. Does the use of orthoses improve self-reported pain and function measures in patients with plantar fasciitis? A meta-analysis. Phys Ther Sport 2009;10:12-18. 
Mänty M, Thinggaard M, Christensen K, Avlund K. Musculoskeletal pain and physical functioning in the oldest old. Eur J Pain 2014;18:522-529.

Metzner G, Dohnalek C, Aigner E. High-energy Extracorporeal ShockWave Therapy (ESWT) for the treatment of chronic plantar fasciitis. Foot Ankle Int 2010;31:790-796.

Pfeffer GB. Plantar heel pain. In: Baxter DE, editor. The foot and ankle sport. St. Louis (MO): Mosby; 1995. p. 195-206.

Pienimaki TT, Tarvainen TK, Siira PT, Vanharanta H. Progressive strengthening and stretching exercises and ultrasound for chronic lateral epicondylitis. Physiotherapy 1996;82:522-530.

Robertson VJ, Baker KG. A review of therapeutic ultrasound: effectiveness studies. Phys Ther 2001;81:1339-1350.

Rompe JD. Shock wave applications in musculoskeletal disorders. Stuttgart (Germany): Thieme; 2002.

Ryan M, Hartwell J, Fraser S, Newsham-West R, Taunton J. Comparison of a physiotherapy program versus dexamethasone injections for plantar fasciopathy in prolonged standing workers: a randomized clinical trial. Clin J Sport Med 2014;24:211-217.

Sahin N, Oztürk A, Atıc1 T. Foot mobility and plantar fascia elasticity in patients with plantar fasciitis. Acta Orthop Traumatol Turc 2010;44: 385-391.

Sems A, Dimeff R, Iannotti JP. Extracorporeal shock wave therapy in the treatment of chronic tendinopathies. J Am Acad Orthop Surg 2006;14: 195-204.

Stasinopoulos D, Johnson MI. Effectiveness of extracorporeal shock wave therapy for tennis elbow (lateral epicondylitis). Br J Sports Med 2005; 39:132-136.

Sweeting D, Parish B, Hooper L, Chester R. The effectiveness of manual stretching in the treatment of plantar heel pain: a systematic review. J Foot Ankle Res 2011;4:19.

Szabó G, Marcsik A, Farkas C. Results of patient information and of a therapeutic exercise program in the treatment of plantar fasciitis. Orv Hetil 2010;151:698-701.

Tounton JE, Ryan BE, Clement DB, Mckenzie DC, Lioyd-Smith DR. Plantar fasciitis; a retrospective analysis of 267 cases. Phys Ther Sport 2002; 3:57-65.

Tsai CT, Chang WD, Lee JP. Effects of short-term treatment with kinesiotaping for plantar fasciitis. J Musculoskelet Pain 2010;18:71-80.

Vahdatpour B, Sajadieh S, Bateni V, Karami M, Sajjadieh H. Extracorporeal shock wave therapy in patients with plantar fasciitis. A randomized, placebo-controlled trial with ultrasonographic and subjective outcome assessments. J Res Med Sci 2012;17:834-838.

Wearing SC, Smeathers JE, Sullivan PM, Yates B, Urry SR, Dubois P. Plantar fasciitis: are pain and fascial thickness associated with arch shape and loading? Phys Ther 2007;87:1002-1008.

Yörük ÖZ, Kırdı N. Extracorporeal shock wave therapy. Med J Suleyman Demirel Univ 2014;2:62-69.

Yüzer S, Sever S, Gürçay E, Ünlü E, Çakcı A. Comparison of the effectiveness of laser therapy and steroid injection in epin calcanei. Turk J Phys Med Rehab 2006;52:68-71. 